\title{
Timely advances in evolving neural-based systems special issue
}

\author{
Lazaros Iliadis $^{1} \cdot$ Konstantinos Margaritis $^{2} \cdot$ Ilias Maglogiannis $^{3}$
}

Received: 17 August 2016 / Accepted: 18 August 2016 / Published online: 25 August 2016

(C) Springer-Verlag Berlin Heidelberg 2016

Professors Plamen Angelov and Nikola Kasabov introduced the term Evolving Systems (EVOS) in 2005 (Angelov and Kasabov 2005, 2006). The definition of this concept serves as an umbrella covering learning approaches that use Soft Computing models that have both their parameters and their structure adapting on-line. Evolving Neuro-Fuzzy Systems are an important chapter of EVOS and the selected papers of this Special Issue fall in this area.

This is the editorial of the "Timely Advances in Evolving Neural-based Systems" Special Issue of the Springer Evolving Systems Journal. It includes selected papers from the 16th EANN 2015 (Engineering Applications of Neural Networks) international conference, organized by the INNS (International Neural Network Society) EANN special interest group. The conference was held in September 2015, in the island of Rhodes Greece.

Initially, eight research papers that were accepted and presented orally in the conference were selected for potential inclusion in the special issue, following high qualitative criteria. Major extensions of these papers were submitted and they passed through a long and careful peer review process. Finally, after two rounds of evaluation, the following

Lazaros Iliadis

liliadis@fmenr.duth.gr

Konstantinos Margaritis

kmarg@uom.gr

Ilias Maglogiannis

imaglo@gmail.com

1 Democritus University of Thrace, Orestiada, Greece

2 University of Macedonia, Thessaloniki, Greece

3 University of Piraeus, Piraeus, Greece six of them were classified as eligible to be included in the Special Issue.

The first paper "Locally Application of Naïve Bayes for Self-Training" was authored by Stamatis Georgios Karlos, Nikos Fazakis, Angeliki-Panagiota Panagopoulou, Sotiris Kotsiantis and Kyrgiakos Sgarbas, from the University of Patras, Greece. They present a novel Self-Labeled Weighted Variant Local Learner, which uses Naïve Bayes as the base classifier of the self-training scheme. A successful comparison with other established semi-supervised classification approaches has been performed, by using standard benchmark datasets.

The second paper is by Athanasios Tsadiras and George Zitopoulos from the Aristotle University of Thessaloniki, Greece. It is entitled "Fuzzy Cognitive Maps as a Decision Support Tool for Container Transport Logistics". It refers to an interesting financial application of Soft Computing, by presenting the employment of Fuzzy Cognitive Maps (an approach that combines Artificial Neural Networks and Fuzzy Logic) towards decision making and management of the Container Transport Logistics.

Peter Hajek and Vladimir Olej from the University of Pardubice in the Czech Republic are presenting an interesting intuitionistic fuzzy inference system (IFIS) in their paper "Intuitionistic Neuro-Fuzzy Network with Evolutionary Adaptation". More specifically they discuss the design of intuitionistic neuro-fuzzy networks to adapt the antecedent and consequent parameters of IFISs. Also they suggest a mean of maximum defuzzification method for a class of Takagi-Sugeno IFISs and compare this approach with the basic defuzzification distribution operator. Moreover regarding the adaptation of the intuitionistic neuro-fuzzy network, they prove that particle swarm optimization provides higher prediction accuracy compared to traditional algorithms, based on gradient descent or least-squares estimation. 
Androniki Tamvakis, Georgios Tsekouras, Anastasios Rigos, Christos Kalloniatis, Christos-Nikolaos Anagnostopoulos, from the University of the Aegean Greece and George Anastassopoulos from the Democritus University of Thrace Greece, have authored the fourth paper. Its title is "A Methodology to Carry Out Voting Classification Tasks Using a Particle Swarm Optimization-Based Neuro-Fuzzy Competitive Learning Network". It discusses the generation of an optimal ensemble of classifiers aiming in the maximization of the classification performance following the voting ensemble method. The classifiers are treated as objects represented by binary vectors and the dissimilarities between pairs of classifiers are quantified. Then, a multidimensional scaling approach is put in place to transform the classifiers into points in a low-dimensional Euclidean space. The set of the resulting points is processed by a neuro-fuzzy competitive learning network trained by a hybrid procedure combining the merits of fuzzy clustering and particle swarm optimization.

The fifth paper is entitled "Adaptive Confidence Learning for the Personalization of Pain Intensity Estimation Systems". The authors are Markus Kächele, Mohammadreza Amirian, Patrick Thiam, Philipp Werner, Steffen Walter, Günther Palm, Friedhelm Schwenker from the University of Ulm, Germany. They discuss a novel method for the continuous estimation of pain intensity, based on fusion of bio-physiological and video features. Additionally, they present a new approach for the adaptation of the system to unknown test persons based on unlabeled data. A large set of features is extracted from the available bio-physiological channels (ECG, EMG and skin conductivity) and the video stream. The authors have managed to show that their algorithm is able to detect samples which can be used to improve the overall performance.

The sixth and last paper is authored by Mario Malcangi and Philip Grew, Universita degli Studi di Milano, Italy and it refers to Speech Recognition. The research is based on the fact that integration of audio and visual data from speech information, improves the performance of an automatic speech recognition system. This can be done by differentiating between the most critical cases of phoneticunit mismatch that occur when processing audio or visual input alone. The evolving fuzzy neural network inference approach and a respective model have been applied at the decision layer, to accomplish this task. The experiments confirm the reliability of the proposed model.

We would very much like to thank the Editors in Chief of the EVOS Journal, Professors P. Angelov, D. Filev, N. $\mathrm{K}$. Kasabov, for their kind support and for giving us the chance to edit this Special Issue.

We hope that we have achieved to offer the International Scientific community a timely view of the Evolving Systems applications in diverse domains and moreover we believe that we have managed to present novel algorithmic approaches adding merit to the existing literature.

The Guest Editors

\section{References}

Angelov P, Kasabov N (2005) Evolving computational intelligence systems. In: Proceedings of the 1st International Workshop on Genetic Fuzzy Systems, Granada, Spain, pp 76-82

Angelov P, Kasabov N (2006) Evolving intelligent systems, eIS, IEEE SMC eNewsLetter, June 2006, pp 1-13 